\title{
Large Scale Reasoning on the Semantic Web: What to Do When Success Is Becoming a Problem
}

\author{
Frank van Harmelen \\ AI Department \\ Vrije Universiteit Amsterdam, The Netherland \\ Frank.van.Harmelen@cs.vu.nl
}

In recent years, the Semantic Web has seen rapid growth in size (many billions of facts and rules are now available) and increasing adoption in many sectors (government, publishing industry, media). This success has brought with it a whole new set of problems: storage, querying and reasoning with billions of facts and rules that are distributed across different locations. The Large Knowledge Collider (LarKC) is providing an infrastructure to solve such problems. LarKC exploits parallelisation, distribution and approximation to enable Semantic Web reasoning at arbitrary scale. In this presentation we will describe the architecture and implementation of the Large Knowledge Collider, we will give data on its current performance, and we will describe a number of use-cases that are deploying LarKC. 